\title{
Estimating the Degree of Nonlinearity in Transient Responses with Zeroed Early-Time Fast Fourier Transforms
}

\author{
Matthew S. Allen \\ Assistant Professor \\ Department of Engineering Physics \\ University of Wisconsin-Madison \\ 535 Engineering Research Building \\ 1500 Engineering Drive \\ Madison, WI 53706-1609 \\ msallen@engr.wisc.edu
}

$\&$

\author{
Randall L. Mayes \\ Distinguished Member of Technical Staff \\ Sandia National Laboratories ${ }^{\star}$ \\ PO Box 5800 \\ Albuquerque, NM 87185 \\ rlmayes@sandia.gov
}

\begin{abstract}
:
This work presents a class of time-frequency methods for detecting and characterizing nonlinearity in transient response measurements. The methods are intended for systems whose response becomes increasingly linear as the response amplitude decays. The discrete Fourier Transform of the response data is found with various sections of the initial response set to zero. These frequency responses, dubbed Zeroed Early-time Fast Fourier Transforms (ZEFFTs), acquire the usual shape of linear Frequency Response Functions (FRFs) as more of the initial nonlinear response is nullified. Hence, nonlinearity is evidenced by a qualitative change in the shape of the ZEFFT as the length of the initial nullified section is varied. These spectra are shown to be sensitive to nonlinearity, revealing its presence even if it is active in only the first few cycles of a response, as may be the case with macro-slip in mechanical joints. They also give insight into the character of the nonlinearity, potentially revealing nonlinear energy transfer between modes or the modal amplitudes below which a system behaves linearly. In some cases one can identify a linear model from the late time, linear response, and use it to reconstruct the response that the system would have executed at previous times if it had been linear. This gives an indication of the severity of the nonlinearity and its effect on the measured response. The methods are demonstrated on both analytical and experimental data from systems with slip or impact nonlinearities.
\end{abstract}

\section{Introduction}

The vast majority of structural dynamic systems are modeled as linear and time invariant, in part because this class of systems is well understood and a number of powerful tools are available for their analysis, so there is often a need to perform experiments to determine whether a given system can be adequately modeled as linear. Since all real systems are nonlinear, this boils down to estimating the error that may be introduced by approximating a nonlinear system as linear. In the event that testing reveals that the system of interest cannot be adequately modeled as linear, one would often also like to identify the nature of the nonlinearity.

\footnotetext{
* Sandia is a multiprogram laboratory operated by Sandia Corporation, a Lockheed Martin Company, for the United States Department of Energy's National Nuclear Security Administration under Contract DE-AC04-94AL85000.
} 
This work is motivated by recent experiences testing and modeling components that are subjected to high level shock loading. A variety of critical space and automotive systems are subjected to these types of loads. These components present quite a challenge in both testing and modeling especially if they contain mechanical joints. One reason for this is that bolted joints in the components may slip due to the high forces that the components experience, so they can no longer be treated as linear at high loads. Macro slip in these joints introduces complex nonlinear response because the force in the joint can be hysteretic in nature, depending not only on the displacement of the joint but on the past history of displacement. Also, the shock force spectrum may have significant amplitude out to high frequencies, so many modes may be important when modeling or testing the component's response to the shock. The objective of this work is to evaluate the degree of nonlinearity induced in a subcomponent's response by shock loading using a small number of measurement points.

Numerous methods have been proposed for detecting nonlinearity and for identifying parametric models of nonlinear systems in the past few decades. Kerschen et al provide a thorough and recent survey of identification methods for nonlinear systems [1]. Many schemes are limited to persistent inputs, such as periodic, broadband random, etc..., but those cannot be used in this application for a number of reasons:

1. The components are designed to withstand shock inputs, so we desire to test them in a shock environment.

2. High-amplitude persistent inputs are much more expensive to apply than high level transient inputs. Models derived through steady state testing may not accurately reproduce the response of the system to shock excitation.

3. The components may be destroyed or degraded by prolonged testing with high level periodic inputs.

A number of methods have been presented that are applicable to transient response data, but each of these has limitations that preclude its use in this application. Some are essentially limited to SDOF or low order systems, such as the restoring force surface method [2, 3], others, such as the NARMAX approach, are limited to cases in which one can postulate a suitable mathematical form for the nonlinearity (e.g. polynomials in the states), limiting the physical insight that one can obtain. The most promising methods for the application of interest involve time-frequency analysis [4] and the Hilbert Transform [5]. The Hilbert Transform can be used to detect the instantaneous frequency and decay envelope of a signal, which are related to the instantaneous stiffness and damping respectively, but it is limited to mono-component or single degree of freedom systems. One potentially solution [6] requires enough sensors to construct a modal filter [7, 8], which precludes its use in this work. Other approaches, such as the Huang-Hilbert Transform (HHT) $[5,9]$ show promise, but are not yet fully developed.

Various types of time-frequency analysis techniques have been proposed, such as the short time Fourier transform (STFT), Choi-Williams expansion, and wavelets. These are problematic in the application of interest because the window functions that they employ minimize the influence of the initial transient, but the slipping nonlinearities in the systems of interest may only be active in the first few cycles. There are also always limitations in the frequency resolution that can be obtained. However, the biggest difference between time-frequency methods and the methods presented here is that the spectra obtained by time-frequency methods have different customary shapes than the frequency response functions that most are used to, so insight gleaned from experience with linear systems cannot be directly applied to understand the nonlinear response.

The method presented in this work utilizes the free response of a system and its Fourier Transform, which have the same mathematical form as the impulse response and frequency response of a linear system. As a result, the spectra are easily interpreted if one is familiar with linear frequency response functions. The method is also capable of handling relatively high order systems; it can be applied to virtually any response. A few tools are presented to aide in interpreting the results, and a metric is presented that describes how important the nonlinearity is to the response.

The following section presents the theory behind the nonlinear detection method. The method is applied to analytical data from a system with slip, contact and cubic nonlinearity in Section 3 . Section 4 presents the results of applying the algorithm to experimental measurements from a real system with a bolted joint. Finally, Section 5 presents some conclusions. 


\section{Proposed Tools and Theoretical Development}

It is well known that many nonlinear systems are increasingly well approximated as linear as their response amplitude diminishes. These systems are the focus of this work. (This certainly does NOT include all important nonlinear systems!) Although nonlinear systems do not have linear modes of vibration, we shall speak of such in the following, and in all cases this should be interpreted to mean the modes of the small-amplitude, linearized system. The free response of the linearized system can be represented as a sum of decaying sinusoids, or complex exponentials, as follows:

$$
h(t)=\sum_{r=1}^{2 N} R_{r} \exp \left(\lambda_{r} t\right)=\sum_{r=1}^{N} R_{r} \exp \left(\lambda_{r} t\right)+R_{r}^{*} \exp \left(\lambda_{r}{ }^{*} t\right)
$$

where $R_{r}$ and $\lambda_{r}$ are the complex residue and eigenvalue respectively, () ${ }^{*}$ denotes complex conjugate and $\mathrm{N}$ is the number of modes active in the response. The typical case of under-damped modes has been assumed, resulting in pairs of complex conjugate eigenvalues $\lambda_{r}$ and residues $R_{r}$. The preceding equation can be expressed in terms of real variables in the following form (which may be more familiar to some readers)

$$
h(t)=\sum_{r=1}^{2 N} \exp \left(-\zeta_{r} \omega_{r} t\right)\left[C_{1 r} \sin \left(\omega_{r} t \sqrt{1-\zeta_{r}^{2}}\right)+C_{2 r} \cos \left(\omega_{r} t \sqrt{1-\zeta_{r}^{2}}\right)\right] .
$$

The Fourier transform of eq. (1) has the same form as the standard pole-residue expression for the Frequency Response of a linear system, except that the definition of the residues is different.

$$
H(\omega)=\sum_{r=1}^{2 N} \frac{R_{r}}{i \omega-\lambda_{r}}=\sum_{r=1}^{N}\left(\frac{R_{r}}{i \omega-\lambda_{r}}+\frac{R_{r}^{*}}{i \omega-\lambda_{r}^{*}}\right)
$$

In the preceding, $R_{r}, C_{1 r}$ and $C_{2 r}$ are determined by the initial position and velocity of the system. These affect only the residues (or modal amplitudes) of the response, so, in the frequency domain, the height of the characteristic peaks may change but the shape of each peak does not. In contrast, the zeros in the spectrum of the free response depend on the coefficients $R_{r}$ and hence on the initial conditions. At large amplitudes, the nonlinear restoring forces distort the response, so it may have a very different form than that described above.

The ZEFFT nonlinear detection algorithm arises from the observation that one could zero the responses in either eq. (1) or (2) above before a certain time, which we denote $t_{z}$ and express the resulting response from $t_{z}$ to an arbitrary time $t$ in exactly the same form as given above although with different constants $\mathrm{R}_{\mathrm{r}}$. Hence, responses for different zero times should have similar characteristic shapes. One can create a set of responses $y_{k}(t)$ from the measured response $y(t)$ by setting

$$
y_{k}(t)=\left\{\begin{array}{cc}
0, & t<\left(t_{z}\right)_{k} \\
y(t), & t \geq\left(t_{z}\right)_{k}
\end{array}\right.
$$

which amounts to assigning a value of zero to the initial response up to the zero time $\left(t_{z}\right)_{k}$. (One could, instead, truncate the portion before $t_{z}$, although this zeroing approach is convenient for some of the following developments.)

The Discrete Fourier Transform (DFT) of each of these responses

$$
Y_{k}(\omega)=\operatorname{DFT}\left\{\mathrm{y}_{\mathrm{k}}(\mathrm{t})\right\}
$$

is then found and the DFTs are compared. These spectra $Y_{k}(\omega)$ are called Zeroed Early-Time FFTs (ZEFFTs) or zeroed FFTs throughout this work. One would expect that responses for early zero times (when the amplitude of the response is large and hence the nonlinearity most active) would have different characteristic shapes than the responses at late times. Nonlinearity can then be detected at early times by observing:

1.) Distortion of the shape of the response around the natural frequencies.

2.) The appearance of frequency content away from the system's low amplitude natural frequencies. The phase of the frequency responses obtained from these truncated time series is modified by the delay caused by zeroing the initial response data. The Fourier Transform of the response after zeroing the data for $\mathrm{t}>\mathrm{t}_{\mathrm{k}}$ has the following form. 


$$
Y_{k}(\omega)=\sum_{r=1}^{2 N} \frac{R_{r} e^{\lambda_{r} t_{k}}}{i \omega-\lambda_{r}} e^{i \omega t_{2}}=\sum_{r=1}^{N}\left(\frac{R_{r} e^{\lambda_{r} t_{k}}}{i \omega-\lambda_{r}}+\frac{\left(R_{r} e^{\lambda_{r} t_{k}}\right)^{*}}{i \omega-\lambda_{r}^{*}}\right) e^{i \omega t_{k}}
$$

The term $\exp \left(i \omega t_{k}\right)$ introduces a linearly increasing phase on the responses and is a result of the well known time shifting property of the Fourier Transform. This can be easily eliminated (and must be if the responses are to be curve fit as described in the next section) by multiplying the frequency response by $\exp \left(-i \omega t_{k}\right)$.

\section{Example:}

The method was applied to experimental data from a $12 \mathrm{in.} \mathrm{long} \mathrm{beam} \mathrm{bolted} \mathrm{at} \mathrm{one} \mathrm{end} \mathrm{to} \mathrm{a} 4.6$ in. long beam, as shown in the schematic in Figure 1. The short beam was struck with an instrumented hammer as indicated in the figure, with an impulse whose peak force was $190 \mathrm{lb}$, and the response was recorded on the $12 \mathrm{in.} \mathrm{long} \mathrm{beam.} \mathrm{The} \mathrm{ZEFFTs} \mathrm{of} \mathrm{the} \mathrm{response} \mathrm{were} \mathrm{created} \mathrm{for} \mathrm{various} \mathrm{zero} \mathrm{times} \mathrm{and}$ are shown in Figure 1. The color of the lines indicates the zero time $\left(t_{z}\right)_{k}$ for that particular ZEFFT, and a legend is shown giving the times, in milliseconds for some of the ZEFFTs. The resonance frequency of the mode in focus seems to increase from about $1380 \mathrm{~Hz}$ to about $1403 \mathrm{~Hz}$ as the response amplitude diminishes, causing the peak for early zero times to broaden. This represents a shift of only $1.5 \%$, yet one can clearly observe that the response does not have the usual shape, which is characteristic of a linear system, at early times. (This could be confirmed by attempting to curve-fit the response at early times, which would reveal that a linear modal model with one mode can not be made to agree with the measurement.)

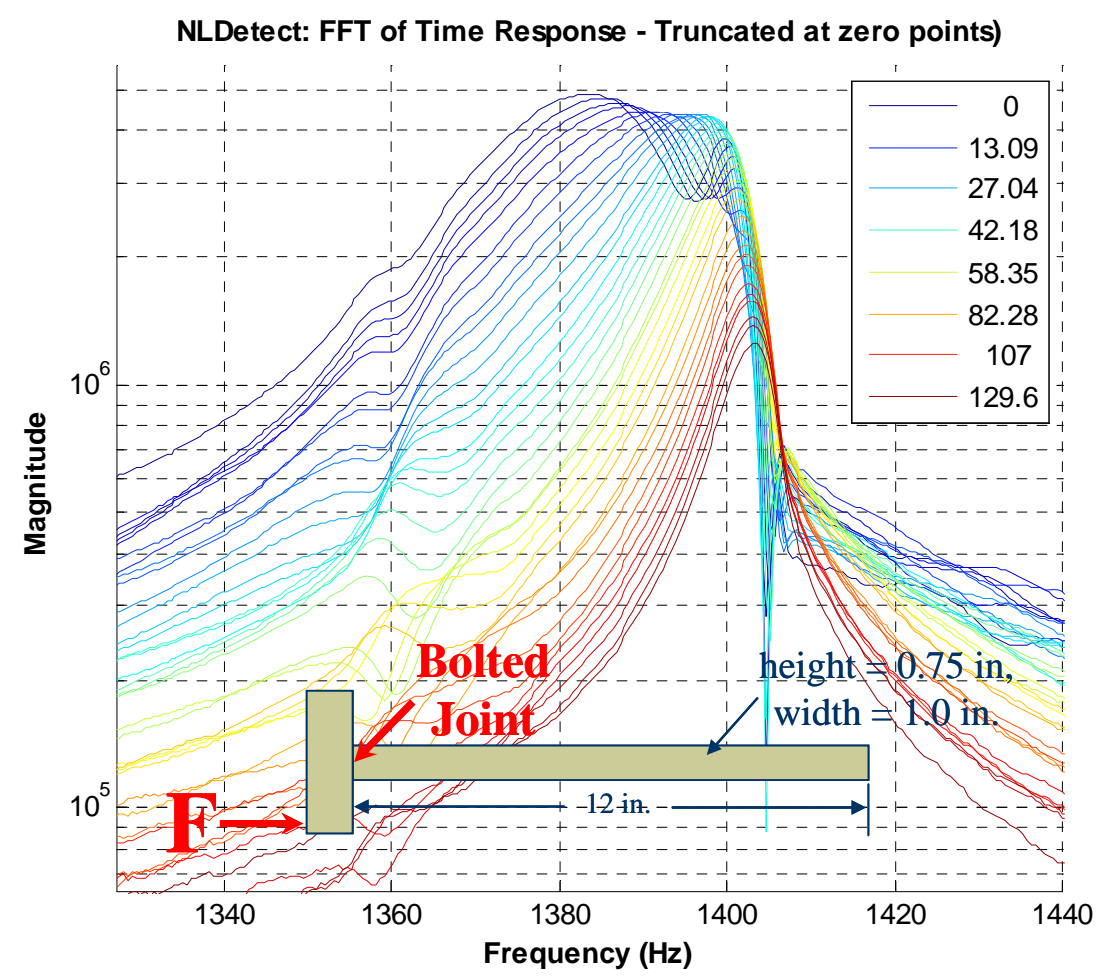

Figure 1: ZEFFTs acquired from a T-beam with a bolted joint. The natural frequency of the mode in focus shifts causing a distortion in the response at early times. This distortion diminishes as the response decays, revealing that the beam is nonlinear and that this linearized mode apparently softens with increasing amplitude. 


\subsection{Backwards Extrapolation for Nonlinearity Detection (BEND)}

In some instances it is not easy to visually discern nonlinear behavior from linear behavior, in part because the zeros of the frequency responses vary with the zero time, complicating the comparisons. This work proposes an approach dubbed Backwards Extrapolation for Nonlinearity Detection (BEND), which aids in visualizing the degree of deviation from linear behavior in a response. The idea is to find a linear approximation to the system at later times (when it is thought to behave linearly) and then propagate that response backwards in time assuming linear behavior. Hence, one can compare the actual response of the system with the response of the linearized fit at each zero time.

The method for achieving this is actually quite simple. If the response after a certain instant, $t_{\mathrm{k}}$, is approximately linear, then the corresponding zeroed response $y_{k}$ can be fit to the form in Equation (1) (this response is zero for $\left.t \leq t_{k}\right)$. This fitting process obtains the poles $\left(\lambda_{r}\right)_{k}$ and residues $\left(R_{r}\right)_{k}$ describing the response for $t>t_{k}$ using one of the methods discussed in the following subsection. Now suppose that we want to propagate this response backwards to time $t_{1}$ in order to compare it with a trace from the original measured response that was truncated at $t_{1}$, where $t_{1}<t_{k}$. Comparing Equations (3) and (6), the residues of the linear system at time $t_{1}$, denoted $\left(R_{r}\right)_{1}$, are related to those at time $t_{2}$, denoted $\left(\mathrm{R}_{\mathrm{r}}\right)_{2}$ as follows:

$$
\left(R_{r}\right)_{2}=\left(R_{r}\right)_{1} e^{\lambda_{r}\left(t_{1}-t_{2}\right)} .
$$

The modal parameters $\left(\lambda_{r}\right)_{1}$ and $\left(R_{r}\right)_{1}$ can be used to reconstruct the linear approximation to response in the time domain using eq. (1), or they can be used to reconstruct the ZEFFT $Y_{k}^{\text {lin }}(\omega)$ using eq. (3) in the frequency domain.

\subsection{Suitable Linear Response Identification Methods}

A variety of methods exist that could be used to identify a linear pole-residue model from the responses at late times. Allemang and Brown [10] showed how most of these can be viewed in a common framework, and texts by Maia et al [11] and Ewins [12] also review a number of methods. There are a few questions that one should consider for the present application.

1.) Does the algorithm return non-physical or computational modes?

2.) How accurately is damping estimated?

3.) Is the system identification algorithm robust to noise?

The first two are important in light of eq. (7), and considering the effect that the damping in each mode has on the extrapolation. Error in the identified damping will directly affect the extrapolation. Some algorithms introduce computational modes that are often either heavily damped or negatively damped [13], and this would clearly have an adverse affect on the extrapolation.

To circumvent these difficulties, the authors have used an iterative system identification algorithm that attempts to identify only the true physical modes of the system. The algorithm, dubbed the Algorithm of Mode Isolation (AMI), was first presented by Drexel and Ginsberg [13] and later extended and validated by Allen and Ginsberg [14-20]. The algorithm works on frequency domain response data by identifying and subtracting modes from the data until it is reduced to noise (care is taken to avoid identifying and subtracting spurious modes), and the modes identified are then refined through an iterative procedure.

\subsection{Single Trace Metric: Integral BEND (IBEND)}

One metric for assessing the degree of nonlinearity of a system as a function of time can be constructed by comparing the total area over a specified frequency band of the difference between the measured and reconstructed magnitude FRF curves. (The reconstructed FRF curves are found by identifying the linear system from the frequency response at a certain zero time and then propagating it forward and backward to all other times.) Let $Y_{k}(\omega)$ and $Y_{k}^{\text {lin }}(\omega)$ denote the measured and linearly extrapolated ZEFFTs associated with zero time $t_{k}$. The nonlinearity metric, $I B\left(t_{k}\right)$, dubbed the Integral of the Backwards Extrapolation for Nonlinear Detection (IBEND), is given by the following, 


$$
I B\left(t_{k}\right)=\frac{\int_{\omega_{L}}^{\omega_{H}}|| Y_{k}(\omega)|-| Y_{k}^{\text {lin }}(\omega) \mid d \omega}{\int_{\omega_{L}}^{\omega_{H}}\left|Y_{k}(\omega)\right| d \omega}
$$

where $\omega_{L}$ and $\omega_{H}$ denote the lower and upper frequency limits over which the integral is computed. The integrals are computed for each zero time using trapezoidal integration. If the response at the time at which the curve fit was performed is truly linear, metric $I B\left(t_{k}\right)$ should be near zero for all times. As the IBEND curve is traced backwards in time, the first time encountered (latest time) at which its magnitude is significantly greater than zero indicates the end of nonlinear behavior. The accuracy of the system identification results can also be verified by observing that the metric remains small at times in which the system is thought to behave linearly. This will be illustrated in the following sections.

\section{Analytical Examples}

The nonlinearity detection algorithm was tested by applying it to synthetic data from the seven degree-of-freedom system shown in Figure 2. The system consists of a small subsystem connected to a large master system through a nonlinear element. Because the attached system is small, the nonlinearity is most evident in the responses of the attached system. All of the masses are constrained to move in the $x$-direction only. The following parameters were used for the system: $m_{1}$ through $m_{5}=1 \mathrm{~kg}, m_{6}=m_{7}$ $=0.1 \mathrm{~kg}, \mathrm{k}_{1}$ through $\mathrm{k}_{4}=1 \mathrm{e} 6 \mathrm{~N} / \mathrm{m}, \mathrm{k}_{6}=1 \mathrm{e} 6 \mathrm{~N} / \mathrm{m}$. Mass and Stiffness proportional damping was used (the nonlinear connection was not included in its calculation) with the following damping matrix: $[\mathrm{C}]=\alpha[\mathrm{M}]+$ $\beta[\mathrm{K}]$ with $\alpha=8, \beta=0.00003$, which gave modal damping ratios for the system (linearized about its static equilibrium) between $1.5 \%$ to $7 \%$. Two different functions were used for the nonlinear restoring force $k_{5}$, as discussed in the following subsections.

The system was excited by applying a half-sine excitation pulse with $0.1 \mathrm{~ms}$ duration and with total energy $E_{\text {in }}$ to mass 5, where the value for $E_{\text {in }}$ was different for each type of nonlinearity, as discussed in the following subsections. To simulate a realistic scenario in the application of interest, it was assumed that only the acceleration of mass $\operatorname{six}\left(\mathrm{d}^{2} \mathrm{x}_{6} / \mathrm{dt}^{2}\right)$ was measured.

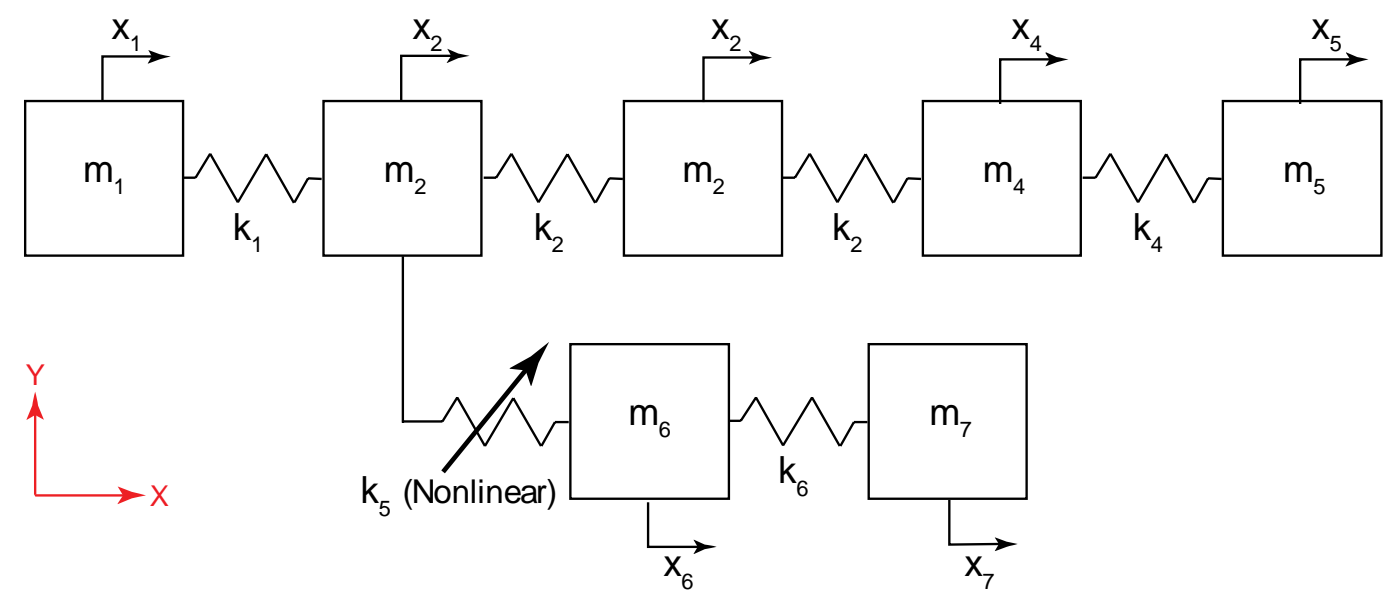

Figure 2: Seven DOF Mass-Spring System with a Nonlinear Connection

The following subsections describe the nonlinear force-displacement laws used for $k_{5}$, specifically: 1.) macro-slip nonlinearity (Jenkins element) and 2.) elastic impact type nonlinearity, and present the results of the proposed algorithms.

\subsection{Slip Nonlinearity}

For this case the connection between masses 2 and 6 was modeled using a Jenkins element [21] with a stiffness of $1 \mathrm{e} 6 \mathrm{~N}$ and a slip force of $1000 \mathrm{~N}$. The excitation energy was $E_{i n}=3 \mathrm{e} 9$ Joules, and the response of the system was found using Euler's time integration method with a massless degree of freedom for the joint displacement. This system will be denoted the slip system or the system with the slip nonlinearity from this point forward. 
Figure 3 shows the acceleration response of mass 6 of the slip system to the impulsive force described previously. Also identified are the zero crossings, which were used as the zero times $\left(t_{z}\right)_{k}$ in eq. (4). One can observe that the system dissipates quite a bit of energy in the first few cycles (before $20 \mathrm{~ms}$ ) following which the response appears to decay exponentially.

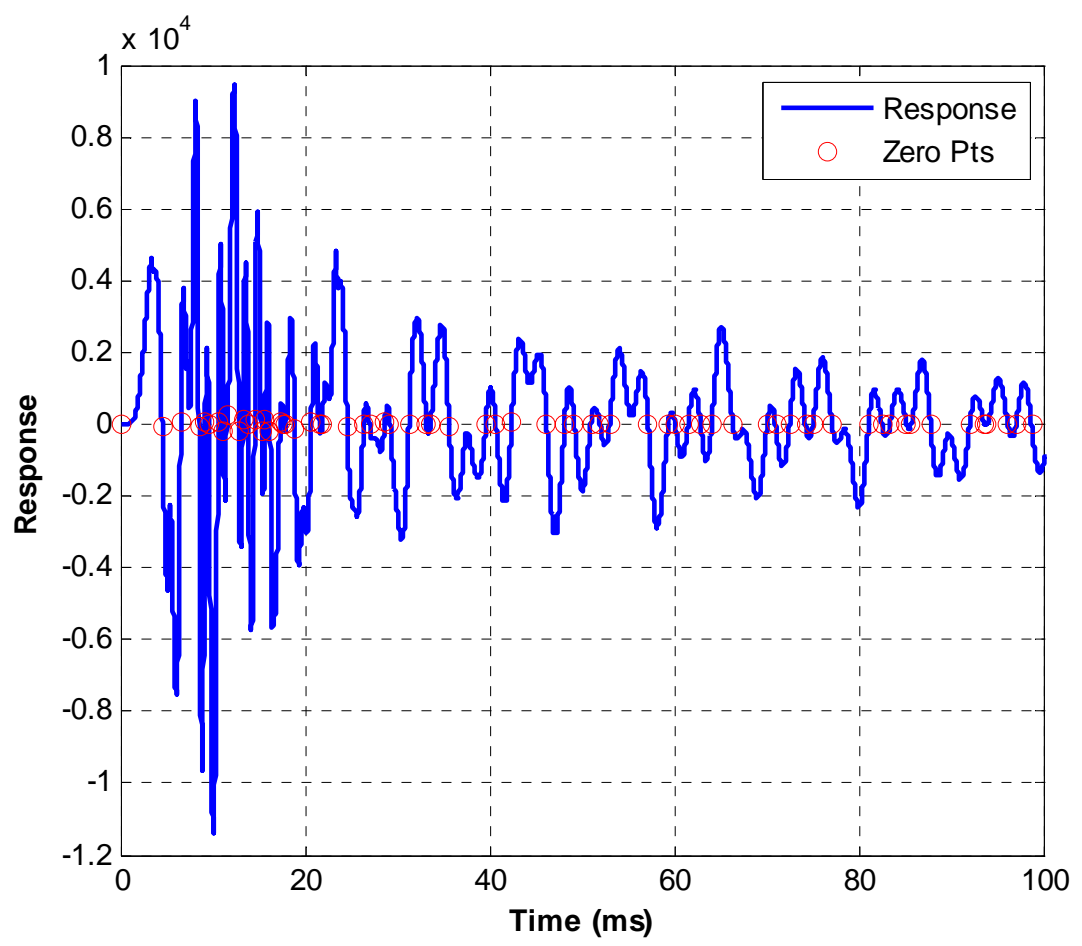

Figure 3: Initial portion of acceleration time response of system with slip nonlinearity. Red circles show the time instants used to create the ZEFFTs.

Figure 4 shows ZEFFTs of the response; one line is shown for each of the times identified with circles in Figure 3. The legend indicates $\left(t_{z}\right)_{k}$ for each response, although some of the legend entries have been omitted for simplicity. The responses exhibit significant changes in character in the 150 to 300 $\mathrm{Hz}$ frequency range for early times (zero times under about 30 milliseconds.) Most notably, the spectrum around the 180 and $230 \mathrm{~Hz}$ modes is lopsided for early zero times and then looks more as expected for a linear system after 17-30ms. 


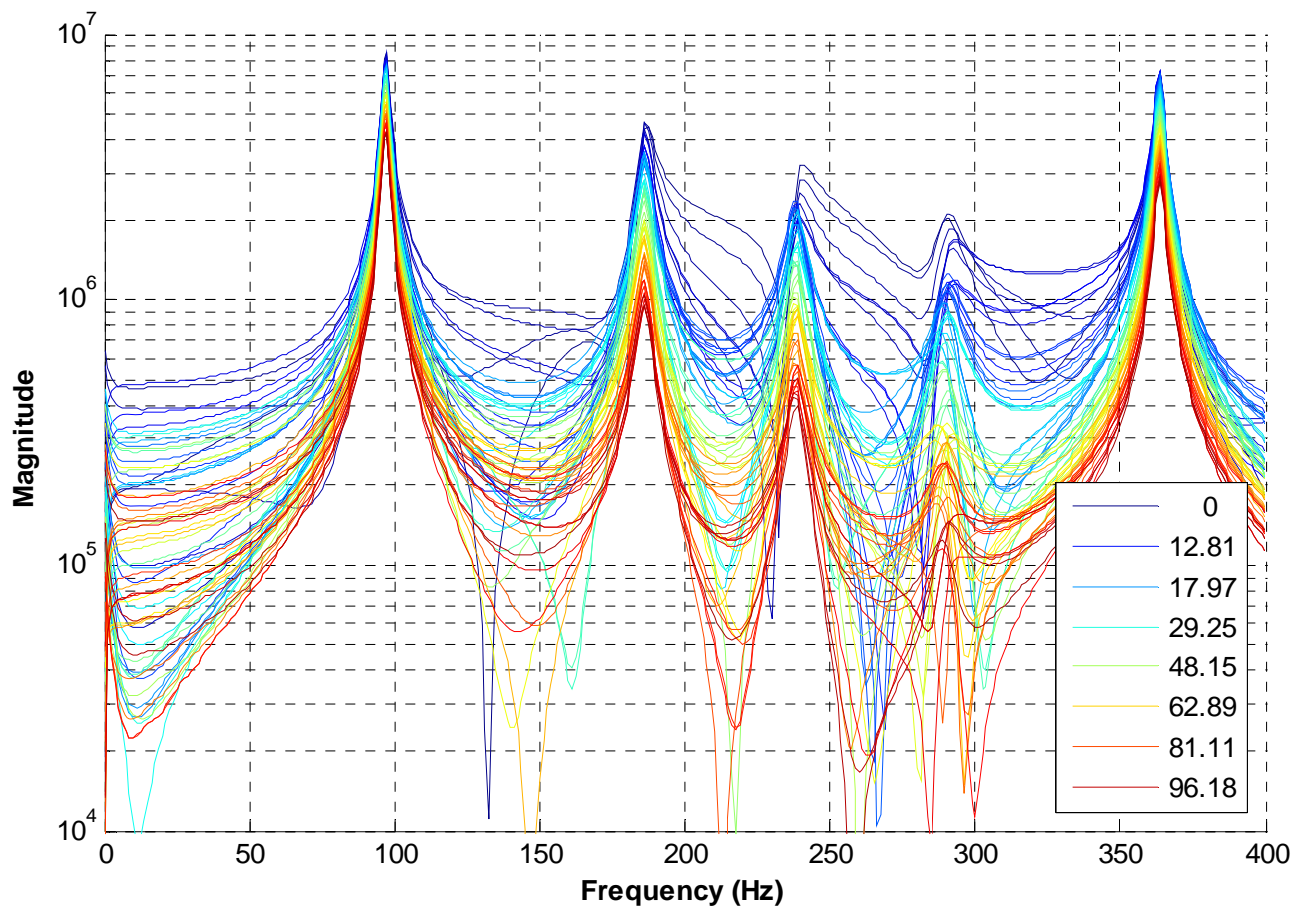

Figure 4: ZEFFTS of Acceleration for slip system for the time response in Figure 3.

Some of the responses in Figure 4 were curve fit and extrapolated backwards in time in order to compare them with the measured (nonlinear) responses from early times. An example of this is shown in Figure 5 where the response at $21.9 \mathrm{~ms}$ was curve fit and was then extrapolated back to $4.52 \mathrm{~ms}$ using eq. (7). (This was the earliest $\left(t_{z}\right)_{k}$ in the set after the input had ceased.) The curve fit at $21.9 \mathrm{~ms}$, shown by a dotted red line, agrees very well to the response data at the same time, shown by a solid red line. However, there are marked differences between the extrapolation of this response and the data at 4.52 ms. First, the response data has much higher amplitude between the resonant peaks than does the linear system extrapolation. Also, the amplitude of the peak near $280 \mathrm{~Hz}$ is considerably lower in the extrapolation than in the measurement. 


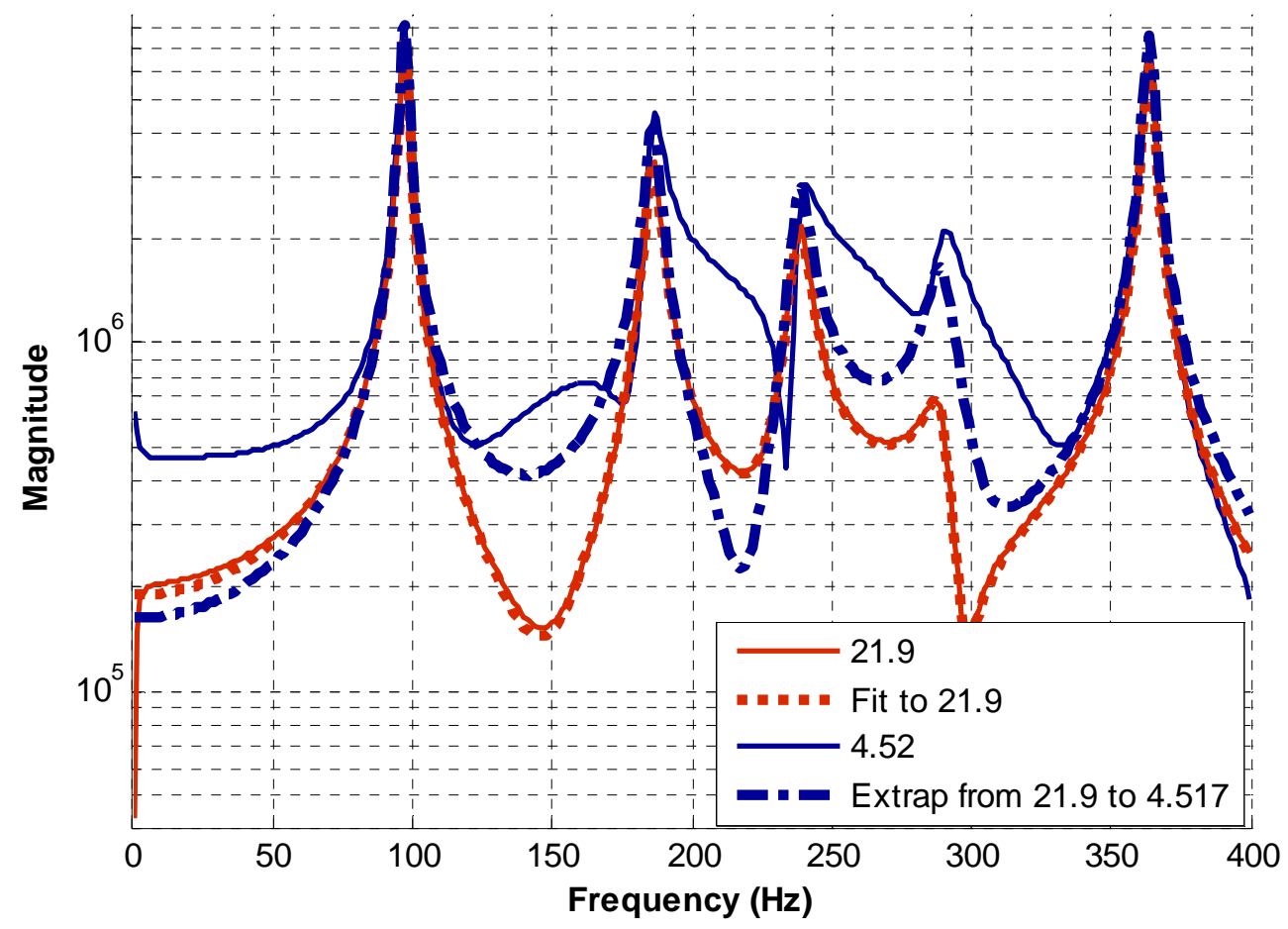

Figure 5: BEND - ZEFFTs of Accelerations for slip system truncated at 0 and 21.9ms, curve fit at $21.9 \mathrm{~ms}$ and extrapolation of the fit to Oms.

The same procedure was repeated with the curve fit applied to the response at $97.1 \mathrm{~ms}$ and then extrapolated to $29.2 \mathrm{~ms}$ (not shown). In this case there was no discernible difference between the 'measured' response and the extrapolated response, suggesting that the system behaved linearly over this time period. The IBEND metric characterizes this over all zero times, and is shown in Figure 6, for the curve fit at $21.9 \mathrm{~ms}$ that was shown in Figure 5. The integral metric is small for all times later than $21.9 \mathrm{~ms}$, suggesting that the curve fit was accurate. Moving backwards in time, one observes that the curves begin to disagree at about 10 or $15 \mathrm{~ms}$, suggesting that the system begins to behave nonlinearly at that time. (Marching forward in time, this is the instant at which nonlinear behavior ceased.)

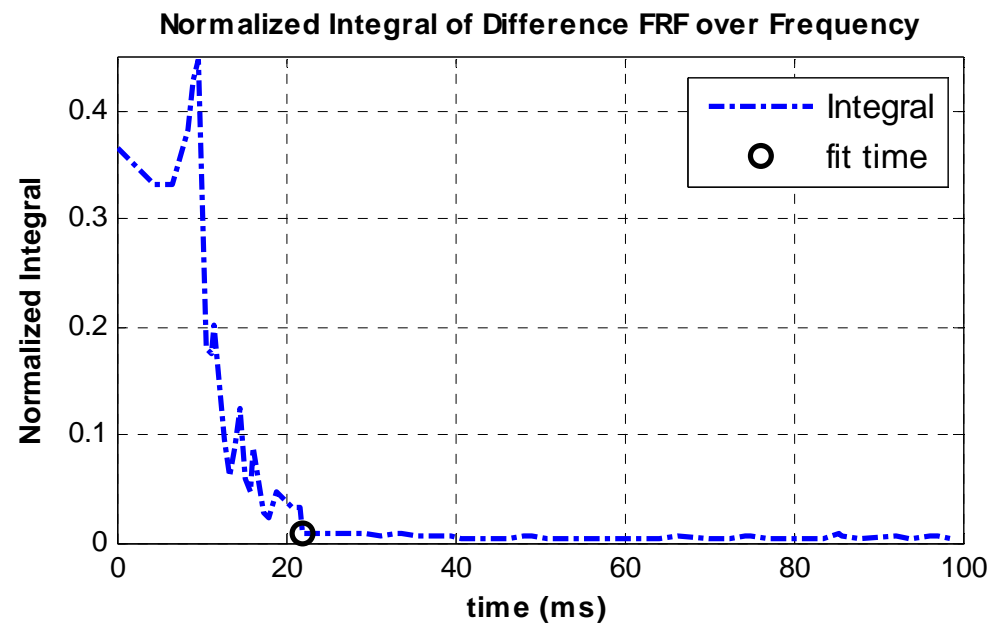

Figure 6: IBEND - metric for system with slip nonlinearity. 


\subsubsection{Discussion}

One interesting feature of Figure 5 is the difference in the heights of the peaks in the measured and extrapolated responses at $280 \mathrm{~Hz}$. The fact that the extrapolated response has lower amplitude than the measured response suggests that this mode dissipated energy due to the nonlinearity before the curve fit was performed (or else transferred it to another part of the spectrum). However, one must use care in interpreting this type of result, because the amplitudes of the peaks in the backwards extrapolation are very sensitive to the damping ratio identified at late times, and damping can be difficult to estimate accurately.

The true nonlinear behavior of the system was found by examining the force in the nonlinear joint versus its displacement, as shown in Figure 7 . The joint slips in two initial cycles all within $12 \mathrm{~ms}$ of the impulse. Following those slip events, the joint behaves almost linearly oscillating between -1 and $-3 \mathrm{~mm}$ displacement. This analytical result validates the insights obtained by the IBEND algorithm. Note that in most experiments a residual displacement such as this would be extremely difficult to measure due to the noise and insensitivity inherent in most transducers at zero frequency.
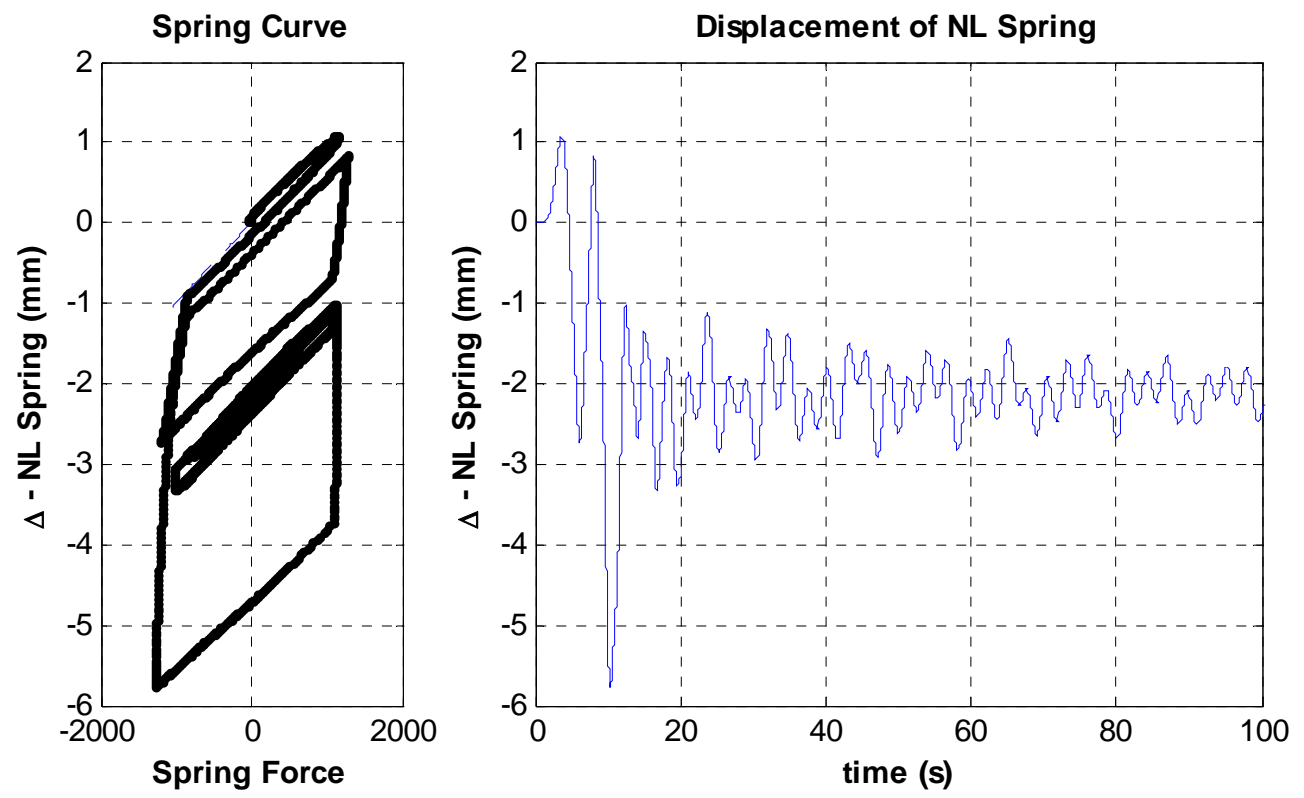

Figure 7: (left) Analytically generated plot of force versus displacement in the slip joint. (right) Displacement of nonlinear joint versus time.

\subsection{Contact Nonlinearity}

This case considers a model for contact nonlinearity in the joint connecting masses 2 and 6 . The following force displacement law was used

$$
f=\left\{\begin{array}{cc}
k_{5} \Delta_{5}+\beta k_{5} \dot{\Delta}_{5} & \Delta_{5}<\Delta_{\text {contact }} \\
k_{c} k_{5} \Delta_{5}+\beta k_{c} k_{5} \dot{\Delta}_{5} & \Delta_{5} \geq \Delta_{\text {contact }}
\end{array}\right.
$$

where $\Delta_{5}=\left(\mathrm{x}_{2}-\mathrm{x}_{6}\right), \mathrm{k}_{5}=1 \mathrm{e} 6 \mathrm{~N} / \mathrm{m}$ and $k_{c}=20$ is a unitless multiplicative factor that specifies how much the stiffness of the joint increases when contact occurs. The excitation energy $E_{\text {in }}$ was $4 \mathrm{e} 9$ Joules.

Figure 8 shows the acceleration of mass 6 for the system with contact nonlinearity. Contact results in large positive accelerations as seen in the early part of the time history. The ZEFFTs were computed and are shown in Figure 9. At early times there is clearly much more spectral energy both at the natural frequencies and throughout the entire frequency band. This energy appears to vanish by about $20 \mathrm{~ms}$. 


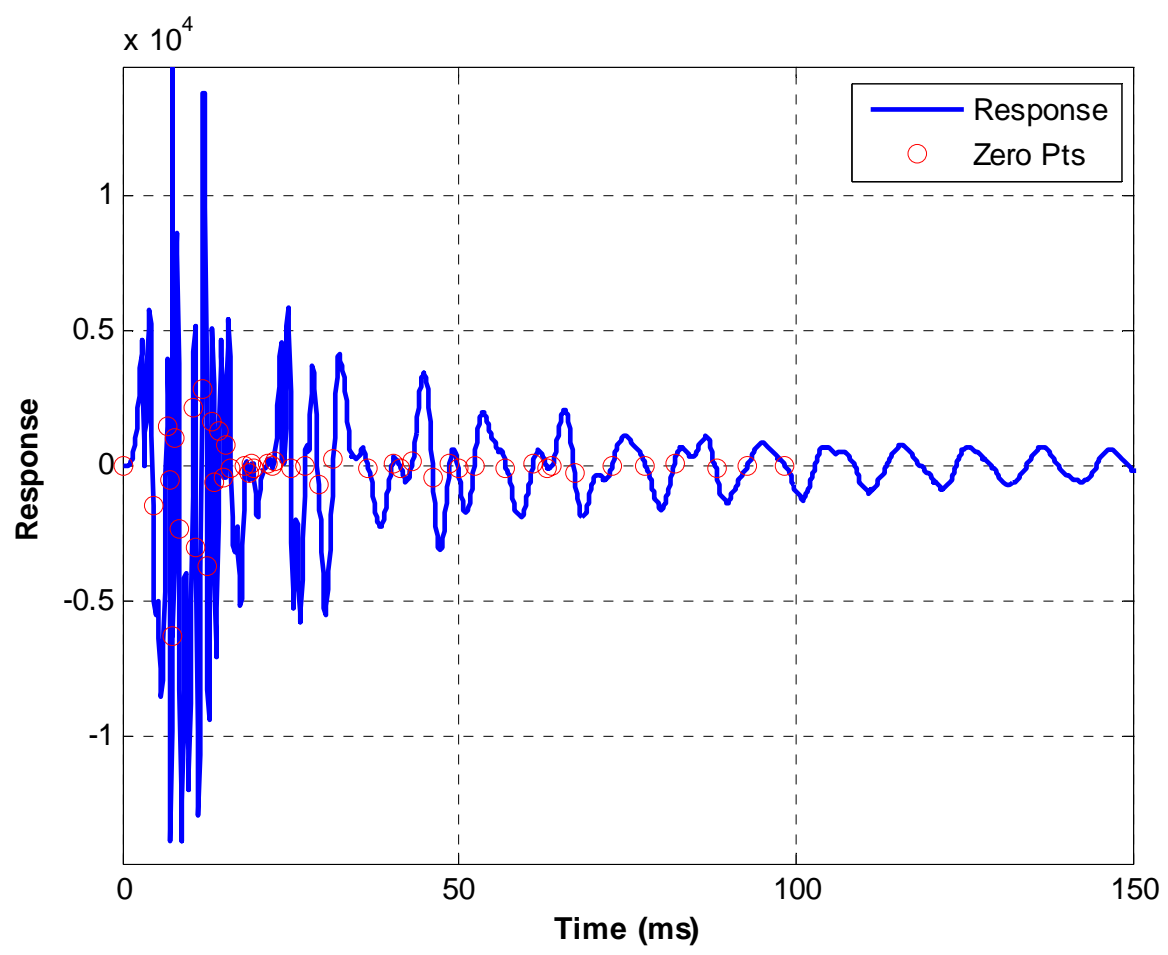

Figure 8: Acceleration time response of system with contact nonlinearity and the time instants used to create the ZEFFTs.

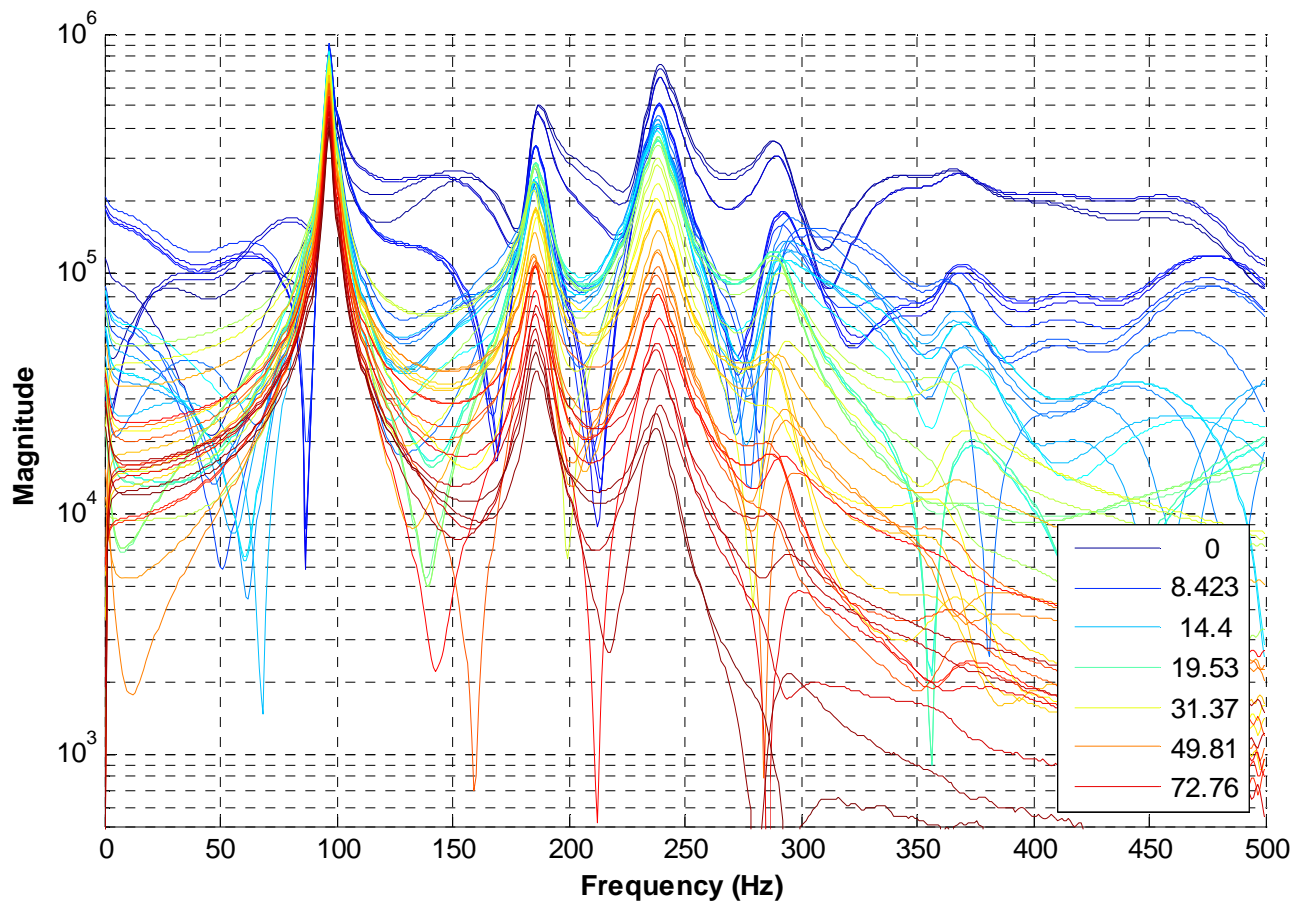

Figure 9: ZEFFTs of Acceleration for contact system, truncated at each of the time instants shown in Figure 8. 
The responses in Figure 9 were curve fit in order to detect the end of nonlinear behavior. It was observed that the 290 and $370 \mathrm{~Hz}$ modes were difficult to identify in this response data. System identification was attempted at a number of zero times before good results were obtained. Figure 10 shows the curve fit to the response at $46.1 \mathrm{~ms}$ and its extrapolation to $4.52 \mathrm{~ms}$. When the identification was performed on earlier ZEFFTs, spurious frequency content above $300 \mathrm{~Hz}$ masked the presence of the $370 \mathrm{~Hz}$ mode and contaminated the damping estimate for the $290 \mathrm{~Hz}$ mode.

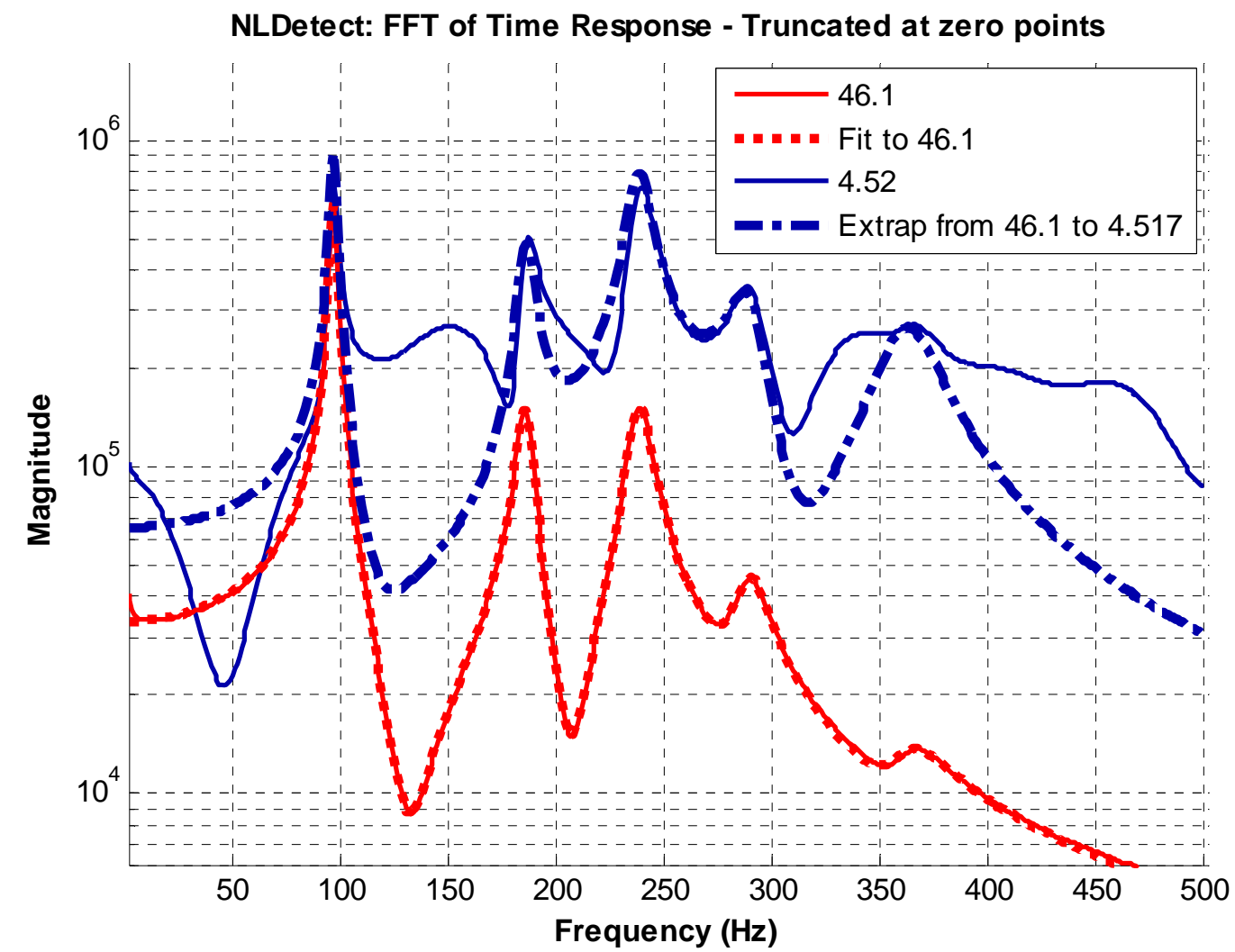

Figure 10: BEND - ZEFFTs of Accelerations for contact system truncated at 0 and $46.1 \mathrm{~ms}$, curve fit at $46.1 \mathrm{~ms}$ and extrapolation of the fit to $0 \mathrm{~ms}$.

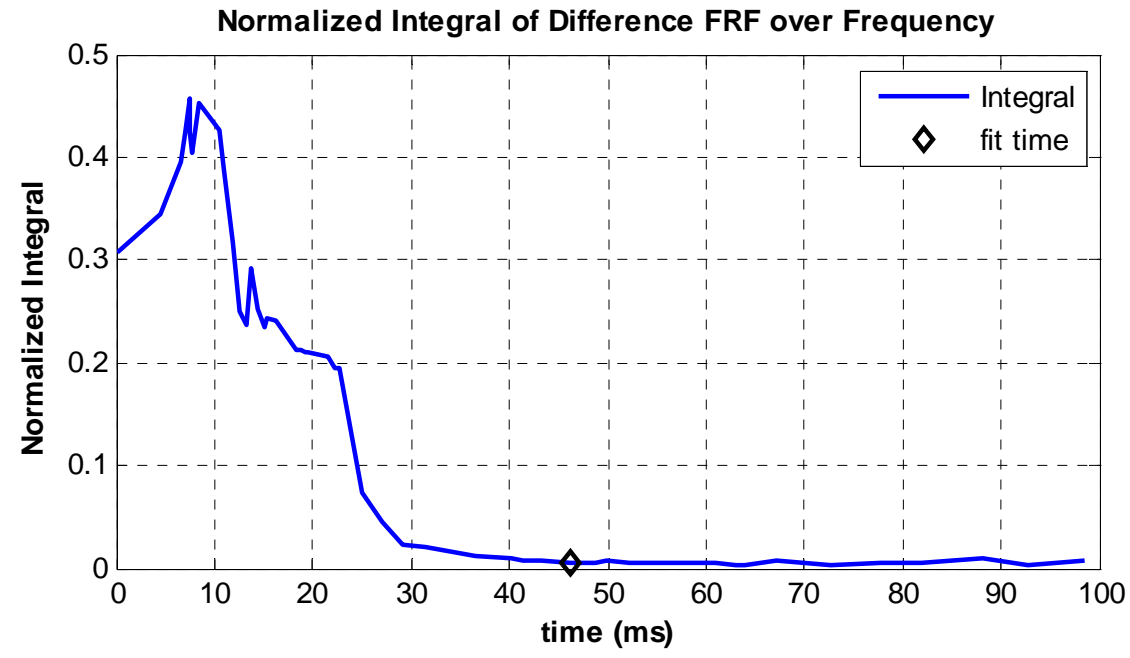

Figure 11: IBEND metric for contact system response data. 
The IBEND metric for this contact system, computed using the ZEFFTs from 0 to $450 \mathrm{~Hz}$, is shown in Figure 11 . The metric becomes small after $25 \mathrm{~ms}$, suggesting that the system is essentially linear for all times after $25 \mathrm{~ms}$.

\subsubsection{Discussion}

Once again, we can confirm these results using the analytical model. Figure 12 shows the displacement in the nonlinear joint both versus time and versus the force in the joint. This indicates that the last contact event occurs just before $25 \mathrm{~ms}$, although the negative amplitude responses suggest that the contacts that occur after $12 \mathrm{~ms}$ are much smaller than those that occur previously.
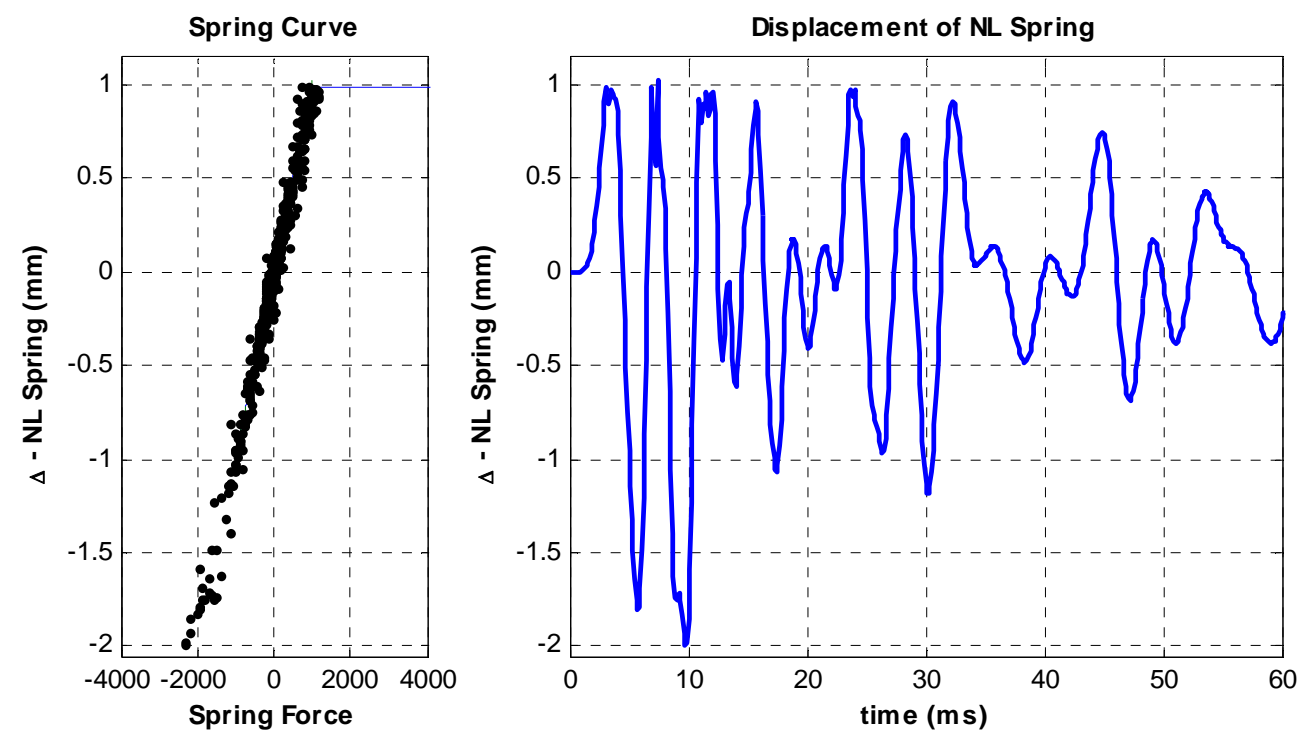

Figure 12: (left) Analytically generated plot of force versus displacement in nonlinear joint for contact system. (right) Displacement of nonlinear joint versus time.

It was noted that there was some difficulty in determining which of the frequency responses in Figure 9 should be used to identify the low-amplitude linear system in this case. In general, one can expect that it may often be necessary to attempt the curve fit at multiple zero times before identifying an appropriate model. During this process, one would like to use late time responses in order to be sure that the nonlinearity is not active, but if data from very late times is used, then quickly decaying modes, may have already fallen below the noise threshold and hence may be difficult or impossible to identify. Fortunately, even in the event that system identification fails so that BEND cannot be applied, one can often gain a good deal of insight by simply inspecting the ZEFFTs.

\section{Experimental Measurements}

This section explores the performance of the nonlinearity detection algorithm on experimental measurements. The data consists of the response of a small component of a larger system that was subjected to impulsive loading. The time response of the system is shown in Figure 13. The nonlinearity detection algorithm was applied at all of the zero crossings indicated in Figure 13, and is shown in Figure 14. A broad peak exists in the early time responses situated between 300 and $600 \mathrm{~Hz}$ that is not typical of a linear system. This peak disappears after about $3.5 \mathrm{~ms}$, revealing a number of sharper peaks between 200 and $600 \mathrm{~Hz}$. 


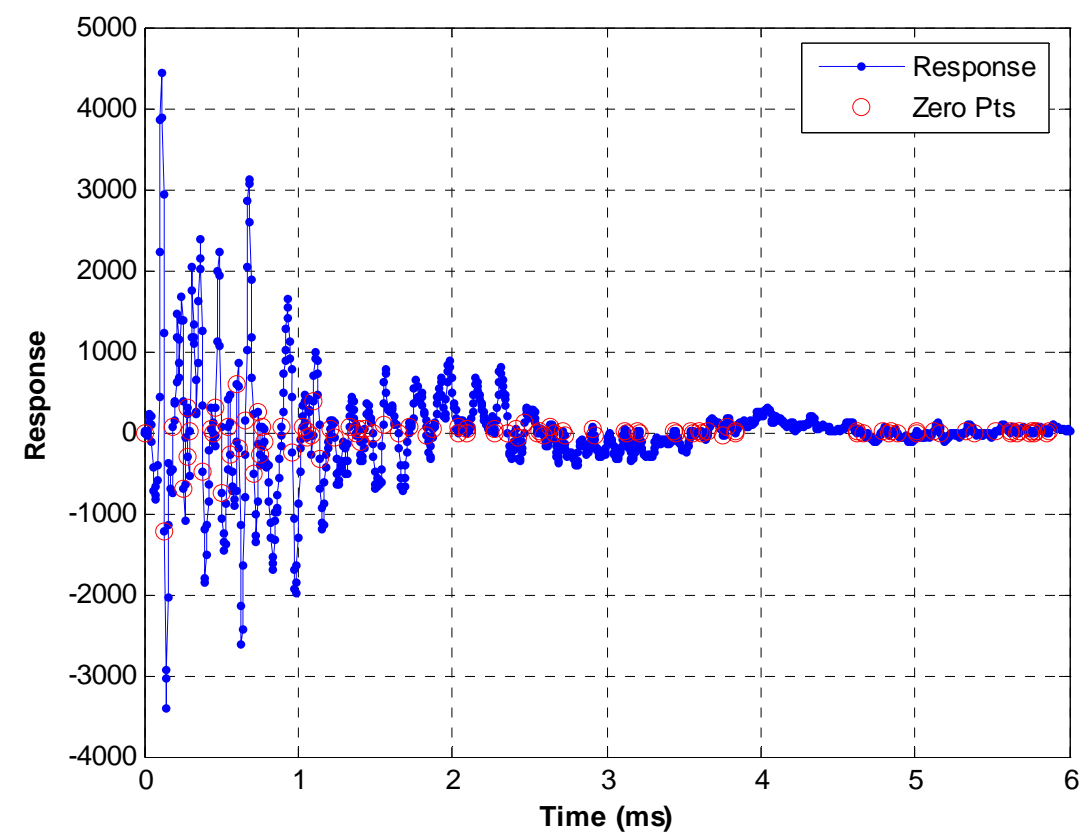

Figure 13: Experimentally measured response on a subcomponent due to an impulsive load.

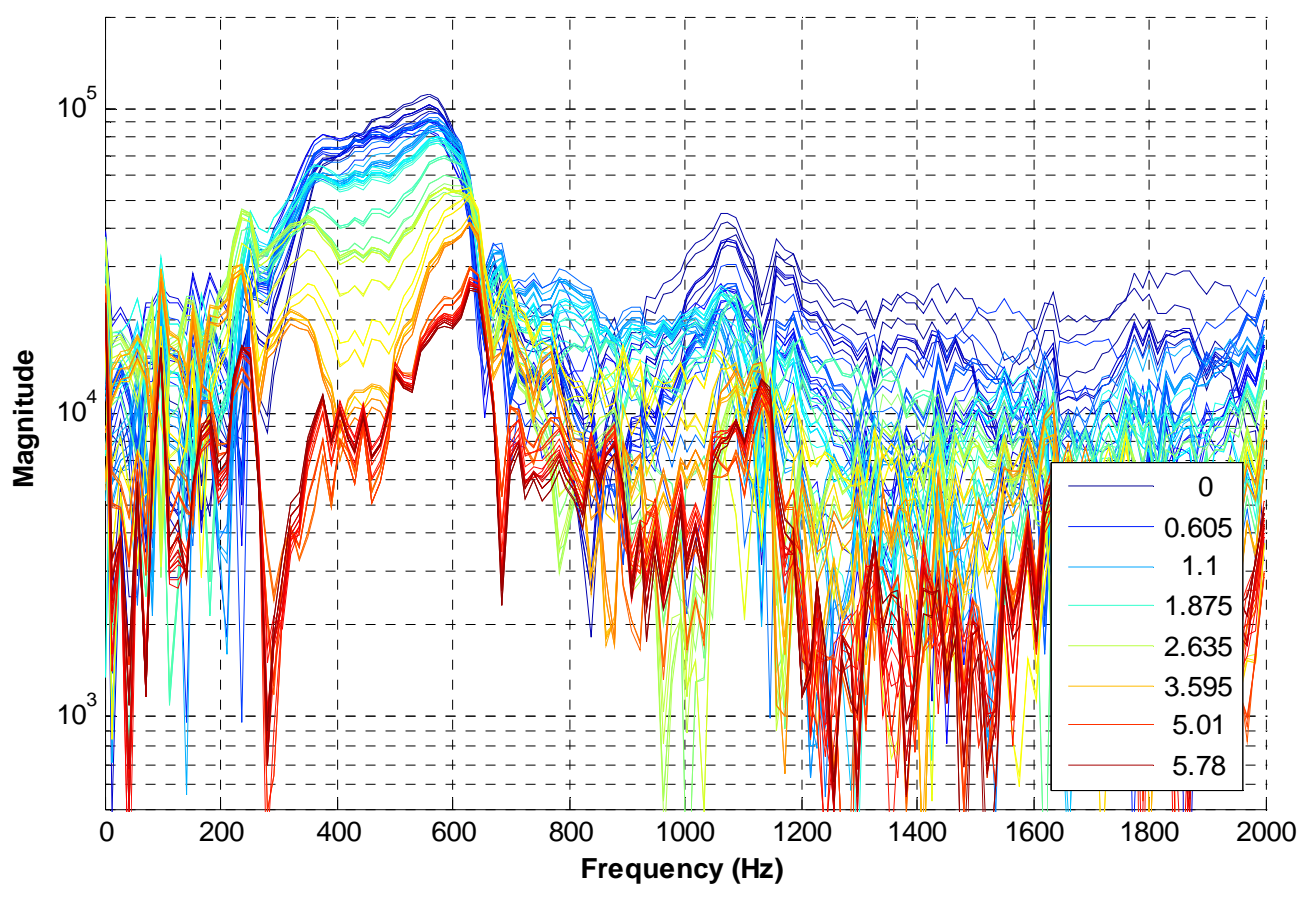

Figure 14: ZEFFTs of experimentally measured acceleration truncated at each of the zero crossings identified in Figure 13.

The AMI system identification algorithm was applied to the ZEFFT of the response at $3.84 \mathrm{~ms}$, identifying four modes below $800 \mathrm{~Hz}$. The curve fit and extrapolations to 0 and $5.87 \mathrm{~ms}$ are shown in Figure 15, along with the corresponding measurements. Good agreement is seen both in the curve fit at $3.84 \mathrm{~ms}$ and its extrapolation to $5.87 \mathrm{~ms}$ below about $675 \mathrm{~Hz}$, suggesting that the curve fit is reasonable 
and that the system behaves linearly over this time period. On the other hand, poor agreement is seen at 0 ms validating the suspicion that the system behaves nonlinearly between 0 and $3.84 \mathrm{~ms}$.

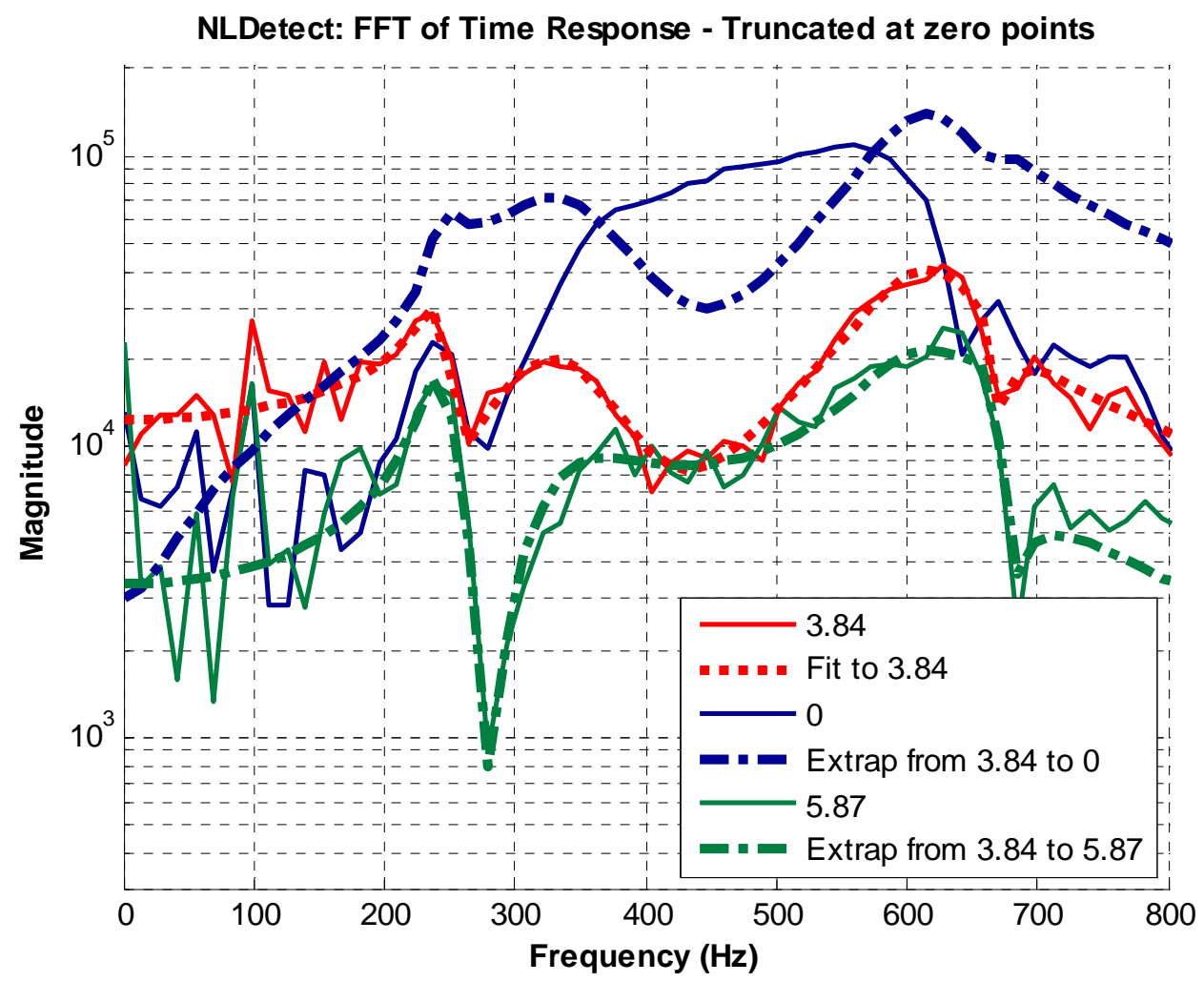

Figure 15: ZEFFTs of experimentally measured accelerations at 0.19, 3.84 and 5.87ms, curve fit at $3.84 \mathrm{~ms}$ and extrapolation of the fit to 0.19 and $5.87 \mathrm{~ms}$.

The IBEND algorithm was applied to the data in the 200 to $650 \mathrm{~Hz}$ frequency band, and is shown in Figure 16. The integral metric suggests that the system behaves linearly after $2 \mathrm{~ms}$. However, the IBEND metric also increases slightly as one marches forward in time from the curve fit instant (3.84 ms), suggesting that the curve fit may not be highly accurate. On the other hand, one can note in Figure 15 that the response at $5.87 \mathrm{~ms}$ is noisier than the $3.84 \mathrm{~ms}$ response that was curve fit, suggesting that noise may have more relative importance to the IBEND metric at late times and hence may cause it to increase artificially. 


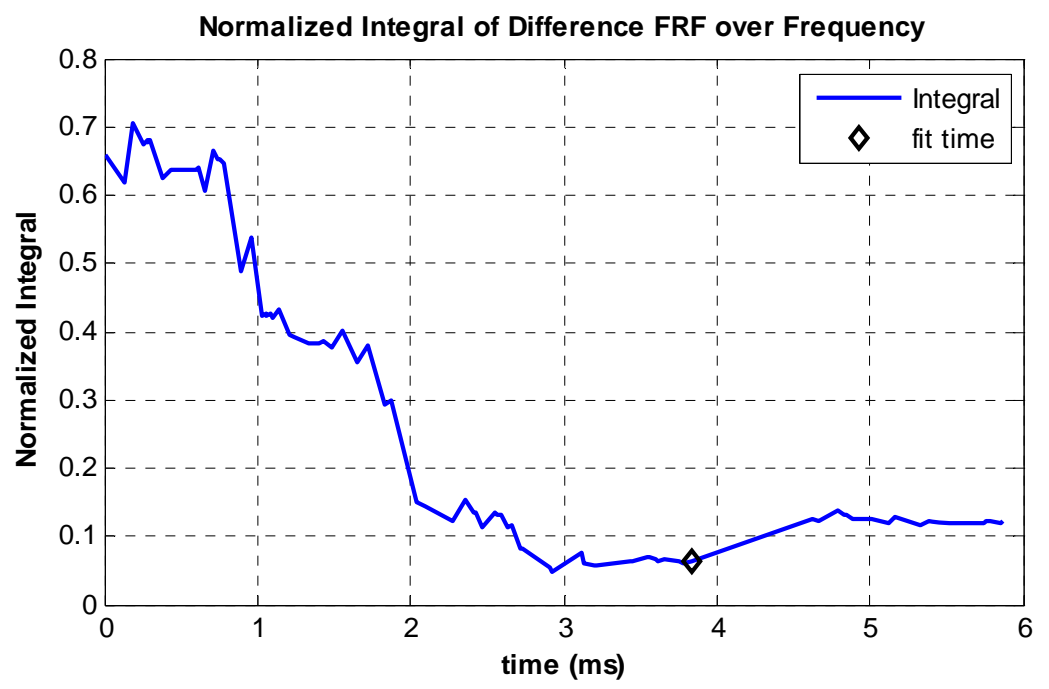

Figure 16: IBEND algorithm for experimental data using 200-650Hz frequency band..

\section{Conclusions}

This work has presented a set of tools that aid in detecting and characterizing nonlinearity in free response data and has evaluated them on both analytical and experimental response data. The first tool dubbed the Zeroed Early-time Fast Fourier Transform (ZEFFT) allows one to interrogate a free response and obtain a visual indication of the degree of nonlinear dynamic behavior in the response. This is done by inspecting the spectra of the response when varying portions of the initial response have been set to zero. When the system response is linear at low amplitude, or during some latter part of its impulse response, one can identify the low amplitude linear system and compare its response with the measured nonlinear response. This was dubbed the Backwards Extrapolation for Nonlinearity Detection or BEND algorithm, and was shown to be useful in clarifying the interpretation of the ZEFFTs. The BEND algorithm can also provide other information. For example, when applied to the analytical slip system the BEND algorithm identified a mode that dissipates a significant amount of energy due to the nonlinear forces. The integral of BEND (IBEND) was shown to provide a single number measure of the degree of nonlinearity in the responses of the systems considered here.

Both analytical and experimental data have been considered in this study. The analytical data illustrated that the algorithm can be used to accurately detect the time (or amplitude level) at which a system begins behaving linearly. This information may be useful when validating analytical models for slip or contact nonlinearity. The method also allows one to evaluate the degree of nonlinearity in the response. Figure 5 showed that the response of the analytical slip system was not very different from its linear approximation while the rest of the systems showed dramatic nonlinear behavior. The algorithm was also applied to experimental data from a system with a bolted joint. This demonstrated that the algorithm is robust to noise and the other limitations inherent to real experiments. The examples presented here have also shown that the algorithm may reveal which modes are most strongly affected by nonlinearity, allowing one to reduce the effort required to perform subsequent nonlinear identification and analysis on the system.

These tools are valuable because they can be applied to relatively high order systems, even if the information obtained is more qualitative than quantitative. The experimental data used in Section 4 came from a system that has tens of modes in the frequency band of interest. However, only a few modes were significant in the response, so system identification was successful in the $200-650 \mathrm{~Hz}$ frequency band. The tools presented here proved very valuable in the test and analysis campaigns of that system, because they provided clear evidence that the system, which was initially thought to be linear, was actually behaving nonlinearly.

\section{Acknowledgements}

This work was performed at Sandia National Laboratories; Sandia is a multiprogram laboratory operated by Sandia Corporation, a Lockheed Martin Company, for the United States Department of 
Energy's National Nuclear Security Administration under Contract DE-AC04-94AL85000. The authors gratefully acknowledge Dan Segalman, Dan Gregory and Todd Simmermacher for their insight and suggestions in developing this work.

\section{References}

[1] G. Kerschen, K. Worden, A. F. Vakakis, and J.-C. Golinval, "Past, present and future of nonlinear system identification in structural dynamics," Mechanical Systems and Signal Processing, vol. 20, pp. 505-592, 2006.

[2] S. F. Masri and T. K. Caughey, "A Nonparametric Identification Technique for Nonlinear Dynamic Problems," Journal of Applied Mechanics, vol. 46, pp. 433-447, 1979.

[3] K. Worden, J. R. Wright, M. A. Al-Hadid, and K. S. Mohammad, "Experimental Identification of Multi Degree-of-freedom Nonlinear Systems using Restoring Force Methods," The International Journal of Analytical and Experimental Modal Analysis, vol. 9, pp. 35-55, 1994.

[4] D. E. Adams and R. J. Allemang, "Survey of Nonlinear Detection and Identification Techniques for Experimental Vibrations," in International Seminar on Modal Analysis (ISMA 23) Leuven, Belgium, 1998, pp. 269-281.

[5] P. F. Pai and J. Hu, "Nonlinear Vibration Characterization by Signal Decomposition," in 24th International Modal Analysis Conference St. Louis, Misssouri: SEM, 2006.

[6] D. Spina, C. Valente, and G. R. Tomlinson, "A New Procedure for Detecting Nonlinearity from Transient Data Using the Gabor Transform," Nonlinear Dynamics, vol. 11, pp. 235-254, 1996.

[7] M. S. Allen and T. G. Carne, "Comparison of Inverse Structural Filter (ISF) and Sum of Weighted Accelerations Technique (SWAT) Time Domain Force Identification Methods," in 47th AIAAASME-ASCE-AHS-ASC Structures, Structural Dynamics, and Materials Conference Newport, RI, 2006.

[8] Q. Zhang, R. J. Allemang, and D. L. Brown, "Modal Filter: Concept and Applications," in 8th International Modal Analysis Conference (IMAC VIII) Kissimmee, Florida, 1990, pp. 487-496.

[9] G. Kerschen, A. F. Vakakis, Y. S. Lee, D. M. McFarland, and L. A. Bergman, "Toward a Fundamental Understanding of the Hilbert-Huang Transform in Nonlinear Structural Dynamics," in 24th International Modal Analysis Conference (IMAC XXIV) St. Louis, Missouri, 2006.

[10] R. J. Allemang and D. L. Brown, "A Unified Matrix Polynomial Approach to Modal Identification," Journal of Sound and Vibration, vol. 211, pp. 301-322, 1998.

[11] S. Maia, J. M. M. Silva, J. He, N. A. Lieven, R. M. Lin, G. W. Skingle, W. M. To, and A. P. V. Urgueira, Theoretical and Experimental Modal Analysis. Taunto, Somerset, England: Research Studies Press Ltd., 1997.

[12] D. J. Ewins, Modal Testing: Theory, Practice and Application. Baldock, England: Research Studies Press, 2000.

[13] M. V. Drexel and J. H. Ginsberg, "Mode Isolation: A New Algorithm for Modal Parameter Identification," Journal of the Acoustical Society of America (JASA), vol. 110, pp. 1371-1378, Sept. 2001.

[14] M. S. Allen and J. H. Ginsberg, "SIMO Extension of the Algorithm of Mode Isolation," in IMAC 22 - XXII International Modal Analysis Conference Dearborn, Michigan, 2004.

[15] M. S. Allen and J. H. Ginsberg, "A Global, Single-Input-Multi-Output (SIMO) Implementation of The Algorithm of Mode Isolation and Applications to Analytical and Experimental Data," Mechanical Systems and Signal Processing, vol. 20, pp. 1090-1111, 2005.

[16] M. S. Allen and J. H. Ginsberg, "Global, Hybrid, MIMO Implementation of the Algorithm of Mode Isolation," in 23rd International Modal Analysis Conference (IMAC XXIII) Orlando, Florida, 2005.

[17] M. S. Allen and J. H. Ginsberg, "Modal Identification of the Z24 Bridge Using MIMO-AMI," in 23rd International Modal Analysis Conference (IMAC XXIII) Orlando, Florida, 2005.

[18] M. S. Allen, "Global and Multi-Input-Multi-Output (MIMO) Extensions of the Algorithm of Mode Isolation (AMI)," in George W. Woodruff School of Mechanical Engineering Atlanta, Georgia: Georgia Institute of Technology, 2005, p. 129.

[19] M. S. Allen and J. H. Ginsberg, "A linear least-squares version of the algorithm of mode isolation for identifying modal properties. Part II: Application and Assessment," Journal of the Acoustical Society of America (JASA), vol. 116, pp. 908-915, 2004. 
[20] J. H. Ginsberg and M. S. Allen, "A linear least-squares version of the algorithm of mode isolation for identifying modal properties. Part I: Conceptual development," Journal of the Acoustical Society of America (JASA), vol. 116, pp. 900-907, 2004.

[21] D. J. Segalman, "A Four-Parameter Iwan Model for Lap-Type Joints," Journal of Applied Mechanics, vol. 72, pp. 752-760, September 2005. 